\title{
Model Experiments on Propagation of Groundwaves Across an Abrupt Boundary at Perpendicular Incidence
}

\author{
R. J. King and S. W. Maley
}

Department of Electrical Engineering, University of Colorado, Boulder, Colo.

(Received June 7, 1965)

\begin{abstract}
An electromagnetic groundwave propagating along the surface of the earth will be partially transmitted and partially reflected at an abrupt electrical discontinuity such as a coastline. An exact theoretical solution for the transmitted and reflected waves appears to be very difficult; however, several investigators have recently found approximated solutions. The accuracy of these solutions has been difficult to evaluate because, when making measurements over the surface of the earth, it is difficult to separate the effects of abrupt electrical discontinuities from those of terrain, nonabrupt electrical discontinuities, etc. To overcome these difficulties a laboratory study has been made using models having carefully controlled dimensions and electrical parameters and having precisely known electrical discontinuities. The laboratory study has shown close agreement with the theory as formulated by J. R. Wait in 1956 and extended recently by the first author in his doctoral thesis.
\end{abstract}

\section{Introduction}

The propagation of electromagnetic groundwaves along nonhomogeneous paths has received considerable interest in the past three decades. As a result, several empirical and semiempirical solutions have been developed and proven useful to a limited extent in calculating the portion of a wave transmitted across a coastline. These methods, generally have been replaced by more rigorous formulations. The theory of groundwave propagation is considered from a tutorial standpoint in two excellent papers by Wait [1962, 1964a], and numerous references are given. The application of accurate theoretical formulations is especially important at low- and verylow frequencies where the earth can be considered as a reasonably good conductor, since these frequencies are widely used in communication and navigation. Experimental results have been difficult to interpret because of the large distances, irregular terrain, and variable ground parameters on mixed propagation paths in actual use.

J. R. Wait has formulated solutions for a number of problems related to groundwave propagation across straight coastlines. He first [Wait, 1956] considered perpendicular propagation across a straight abrupt boundary separating two media which constituted a flat earth. These results were later extended to propagation over a spherical earth, and to paths with more than two sections [Wait and Householder, 1957; Wait, 1961]. Using a scalar approximation, Furutsu [1955, 1956] also considered groundwave propagation over mixed paths, and later [Furutsu, 1963] extended his results to include abrupt changes in elevation. Unfortunately, however, his results are not valid near the separation boundaries.
In four recent papers, Wait and coauthors have derived approximate expressions for the field near a boundary with various properties which is located in the far zone of the transmitting antenna [W ait, 1963, Part I; Wait and Jackson, 1963, Part II; Wait, 1964b, Part III; Wait and Spies, 1964]. Part I describes the vertical electric field near an abrupt boundary, Part II applies to a coastline with a sloping beach and Part III pertains to a beach (transition zone) over which the magnitude of the surface impedance changes linearly from that of one medium to that of the other medium. The paper by $\mathrm{W}$ ait and Spies pertains to a transition zone over which the magnitude of the surface impedance changes gradually and continuously according to a prescribed function.

Theoretical formulations of scientific phenomena are not always completely reliable, and they must be experimentally evaluated. Carefully performed experiments can provide justification of any assumptions which were made, can show the applicability of the theory to special cases, and also the accuracy which the theory furnishes. It is seldom possible to formulate a theoretical problem assuming the true nature of things. In this study a "theoretical model" which approaches the true situation is used. This provides a more tractable mathematical problem, but it is indeed rare when further simplifying assumptions are not necessary.

The logical procedure would then be to use experimental models which simulate the mathematical models as closely as possible if the theoretical solutions are to be verified to any reasonable degree of accuracy. This procedure generally avoids unwanted effects of other nearby terrain and objects. The experiments described here have utilized a small laboratory model which simulates, as closely as possible, 
the mathematical models used in the theoretical formulations. In this way, distances and other parameters can be carefully controlled and measured. Then, if the experimental results compare favorably with the theoretical solutions, the theory can be applied to naturally occurring mixed paths with considerably more confidence.

The most recent experimental results for groundwave propagation were published by Maley and Ottesen [1964]. They verified Wait's [1956] first order result for propagation across a straight abrupt coastline at normal incidence. Measurements were made on a laboratory model very similar to the one described here. The frequency used was $10 \mathrm{Gc} / \mathrm{s}$. The imperfectly conducting medium was ordinary tap water (corresponding to earth at LF and VLF frequencies) and an aluminum sheet was used as a highly conducting medium (sea). The transmitting antenna was a short base-fed monopole and the receiving antenna was an open-ended waveguide which was mounted on a wooden rod and could be moved over the aluminum for various locations of the boundary with respect to the transmitting antenna. The experimental techniques used did not permit accurate measurements of the field at or in front (on the transmitter side) of the boundary. The investigations reported here utilized superior experimental methods and produced data valid in regions both close to and far from the boundary.

\section{Theory}

The general formulation for groundwave propagation in the presence of a mixed path has been given by Wait [1956]. The geometry of the mathematical model is shown in figure 1 . The starting point involves an integral relation obtained from the application of the electromagnetic "compensation theorem." The medium to the right of the coastline is regarded as a perturbation upon a homogeneous earth of surface impedance $Z$. The perturbation of surface impedance from $Z$ to $Z_{1}$ brings about a change of the mutual impedance, from $Z_{m}$ to $Z_{m}^{\prime}$ (let $Z_{m}^{\prime}-Z_{m}$ $=\triangle Z_{m}$ ) between two vertical Hertzian dipoles $A$ and $B$. When $A$ and $B$ are located on a flat ground, the resulting general expression for $\triangle Z_{m}$ is [W ait, 1956]

$$
\begin{gathered}
\Delta Z_{m}=\frac{l_{A} l_{B}}{\lambda^{2}} \int_{x=0}^{+\infty} \int_{y=-\infty}^{+\infty}\left(Z-Z_{1}\right) \frac{e^{-i k(r+R)}}{r R} F(r, Z) F^{\prime}\left(R, Z_{1}, Z\right) \\
\left(1-\frac{i}{k r}\right)\left(1-\frac{i}{k R}\right) \cos \delta d x d y .
\end{gathered}
$$

The lengths of the transmitting and receiving dipoles are $l_{A}$ and $l_{B}$ respectively. The $R$ and $r$ are distances defined in figure $1, k=2 \pi / \lambda$ and $\lambda$ is the free space wavelength. An exp $(i \omega t)$ time factor $(i=\sqrt{-1})$ and the rationalized MKS system of units are used throughout. The $F(r, Z)$ is Sommerfeld's groundwave attenua-

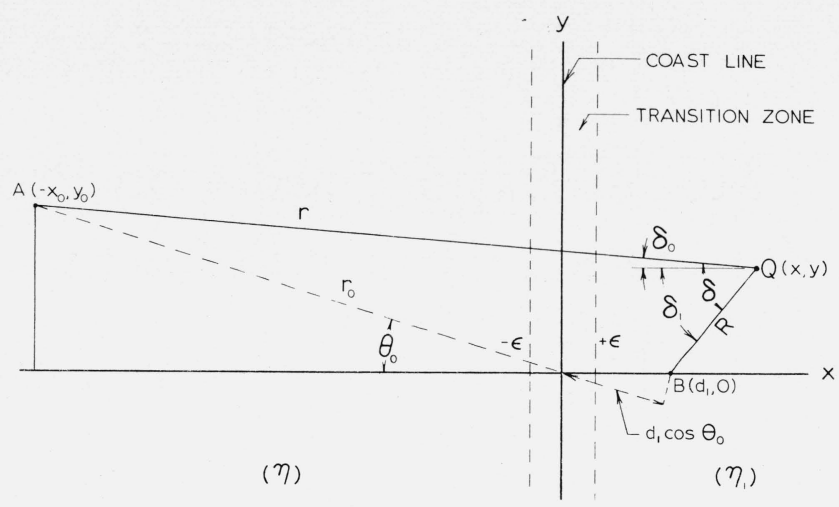

FIGURE 1. Geometry of mathematical model used in the formulation of the problem for oblique propagation of a groundwave across a coastline.

tion function for a homogeneous flat ground defined by

$$
F(r, Z)=1-i \sqrt{\pi p} e^{-p} \operatorname{erfc}(i \sqrt{p})
$$

where $p$ is the "numerical distance" given by

$$
p=-i \frac{k r}{2}\left(\frac{Z}{\eta_{0}}\right)^{2}
$$

and $\eta_{0} \cong 120 \pi$. The surface impedance for grazing incidence $Z$, is related to the intrinsic impedance of the ground,

$$
\eta=\left(\frac{i \omega \mu_{0}}{\sigma+i \omega \epsilon}\right)^{1 / 2}, \text { by } Z=\frac{\eta}{\eta_{0}} \sqrt{\eta_{0}^{2}-\eta^{2}}
$$

a similar equation applies when $Z$ and $\eta$ are replaced by $Z_{1}$ and $\eta_{1}$. The $F^{\prime}\left(R, Z_{1}, Z\right)$ is an unknown attenuation function for transmission from antenna B a distance $R$ for the configuration of ground parameters shown in figure 1 . The permeability of the ground is assumed to be that of free space, $\mu_{0}$.

When calculating the vertical electric field near the boundary for perpendicular incidence, a number of successive approximations can be made in (1). The basic assumptions are that the boundary is many wavelengths from the source, and the receiving antenna is within a wavelength or so of the boundary. Then $k r>>1, F(r, Z) \cong F\left(r_{0}, Z\right), F^{\prime}\left(R, Z_{1}, Z\right) \cong 1$, and, for the case of an abrupt boundary, (1) can be integrated in closed form to obtain the final result [Wait, 1963, Part I]

$$
\frac{\Delta Z_{m}}{Z_{m}} \cong \frac{\Delta_{0}}{2 C_{1}} e^{i \frac{3 \pi}{4}}\left[\left(C_{1}^{2}-i \alpha_{1}\right) H_{0}^{(2)}\left(\left|\alpha_{1}\right|\right)-\left|\alpha_{1}\right| H_{1}^{(2)}\left(\left|\alpha_{1}\right|\right)\right] e^{i \alpha_{1}},
$$

where

$$
\Delta_{0}=\frac{Z-Z_{1}}{\eta_{0}} e^{-i \frac{\pi}{4}}, C_{1}=\cos \theta_{0}, \text { and } \alpha_{1}=k C_{1} d_{1} .
$$


Under the assumptions already made, the essential requirement is that $\left|\Delta Z_{m}\right| Z_{m} \mid<<1$. Then

$$
F^{\prime}\left(d, Z, Z_{1}\right) \cong F(d, Z)\left[1+\frac{\Delta Z_{m}}{Z_{m}}\right]
$$

where $d=r_{0}+C_{1} d_{1}$, and $F^{\prime}\left(d, Z, Z_{1}\right)$ is the unknown attenuation function for transmission between antenna $\mathrm{A}$ and antenna B along the mixed path. Equation (5) can be used to find the electromagnetic fields near the boundary, e.g., the vertical electric field at $\left(d_{1}, 0\right)$ due to a driving current $I_{A}$ in antenna $\mathrm{A}$ is approximately

$$
E^{\prime} \cong-i \frac{l_{A}}{\lambda} I_{A} \eta_{0} \frac{e^{-i k d}}{d} F^{\prime}\left(d, Z, Z_{1}\right)
$$

For a theoretical formulation valid at greater distances from the boundary, it can be assumed that $k r>>1$ and $k R>>1$ in (1). This formulation is given in elliptic coordinates King, [1965b] which parallels the work of Wait [1956] in Cartesian coordinates. The two-dimensional integral was reduced to one dimension by using a stationary phase approximation, and assumes that neither antenna is near a boundary. For the particular case where propagation is over an abrupt hyperbolic boundary at normal incidence (see fig. 2), the approximate solution is

$F^{\prime}\left(d, Z, Z_{1}\right) \cong F(d, Z)$

$$
+\left(\frac{i d}{\lambda}\right)^{1 / 2} \int_{0}^{\theta_{1}}\left(\frac{Z-Z_{1}}{\eta_{0}}\right) F(r, Z) F^{\prime}\left(R, Z_{1}, Z\right) d \theta,
$$

where

$$
\theta_{1}=\cos ^{-1}\left(\frac{2 r_{0}}{d}-1\right)
$$

and $d$ again is the distance between the transmitter and receiver as shown in figure 2 .

Although (7) is valid when A and B are located at the foci of the elliptic coordinate system, it is also nearly valid for a straight abrupt boundary defined by the line $x=x_{1}$ in figure 2 . This is true since the line $x=x_{1}$ is nearly coincident with the hyperbola $\theta=\theta_{1}$ in the vicinity of the connecting line $(\mu=0)$ between antennas $\mathrm{A}$ and $\mathrm{B}$, and propagation takes place mainly along this line. If this assumption is made and then (7) is transformed to a Cartesian coordinate system with its origin at the point $\mu=0$ and $\theta=\theta_{1}$, the same result as that given by $\mathrm{W}$ ait [1956] is obtained:

$F^{\prime}\left(d, Z, Z_{1}\right)=F(d, Z)$

$+\left(\frac{i d}{\lambda}\right)^{1 / 2} \int_{0}^{R_{0}}\left(\frac{Z-Z_{1}}{\eta_{0}}\right) \frac{F\left(r_{0}+\hat{x}, Z\right) F^{\prime}\left(R_{0}-\hat{x}, Z_{1}, Z\right) d \hat{x}}{\left[\left(r_{0}+\hat{x}\right)\left(R_{0}-\hat{x}\right)\right]^{1 / 2}}$.

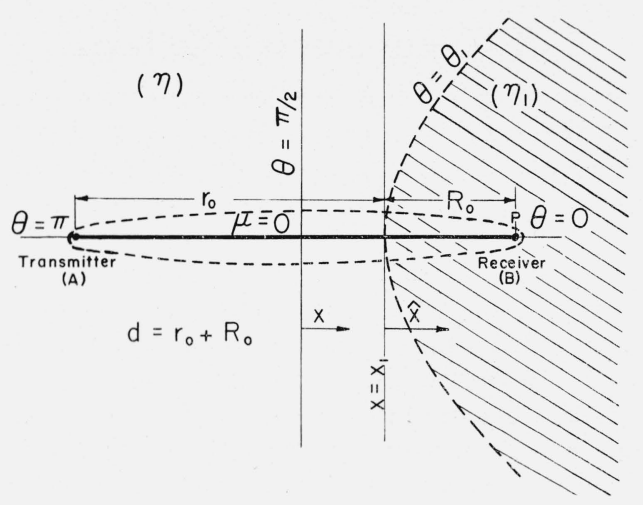

Figure 2. Top view of the elliptic coordinate system on a flat earth.

The perturbation within $S$ has been taken as a hyperbola $0 \leqslant \theta \leqslant \theta_{1}<\pi$, and antennas $\mathrm{A}$ and $\mathrm{B}$ are located at the foci. A straight abrupt coastline indicated by $x=x_{1}$ is approximated by the hyperbola $\theta=\theta_{1}$.

Equations (7) and (8) are nonhomogeneous integral equations in which a good zero-order approximation for the unknown function $F^{\prime}\left(R, Z_{1}, Z\right)$ in the integrand is $F\left(R, Z_{1}\right)$ since the character of the medium behind the observation point has very little effect upon the field at the observation point. Then inserting this function into (7) or (8) yields a first-order solution for $F^{\prime}\left(d, Z, Z_{1}\right)$. Further iteration is possible but not necessary.

Equations (7) and (8) were evaluated using numerical methods and a digital computer. The technique has been fully described by King [1965b]. In general, it was concluded that the convergence of (7) upon successive iteration is quite rapid, and the secondorder results differed from the first by less than 0.6 percent for the parameters used in the experimental model. The numerical integration was accomplished through the use of 8-point Gaussian Quadrature which gave good accuracy and allowed the use of a minimal number of subdivisions. The results checked within six significant figures of the results using 24point Gaussian Quadrature. Equation (8) was programmed in a similar fashion using 24-point Gaussian Quadrature and it was found that convergence upon successive iteration was not as rapid as that of (7), the second-order results being within 5 percent of the first and third-order results were within 1 percent of the second. The convergence of (8) was nearly independent of the number of subdivisions used in the integration procedure, while the integrable singularity in the denominator required a more accurate program. Wait and Householder [1957] avoided the problem presented by this singularity in their numerical calculations by making the change of variable $\hat{x}=R_{0}-u^{2}$ in (8).

For the above reasons, and the fact that (7) is simpler to evaluate, it was used exclusively when calculating the theoretical field as being a free space wavelength or more behind the boundary. Equation (4) was used when calculating the field near or in front of the boundary. 


\section{Experimental Methods}

The frequency employed in the experimental models was $4.2937 \mathrm{Gc} / \mathrm{s}(\lambda \cong 7 \mathrm{~cm})$. Water was used to simulate imperfectly conducting media (complex dielectric constant $=\boldsymbol{\epsilon}_{r}=78.7-\mathrm{il} 3$ ) while aluminum plates simulated perfectly conducting media. Thus, there was a marked contrast in surface impedance between the two sections of the path. The water was contained in a large $(4.88 \mathrm{~m} \times 4.88 \mathrm{~m})$ tank and the depth of water was normally about $7.5 \mathrm{~cm}$ or five skin depths. The mixed path model was surrounded on three sides and above by microwave absorbing material to prevent reflections from interfering with the measurements.

The measurements of the vertical electric field over the mixed paths were made by a modulated scattering dipole technique and a specially designed microwave receiver described elsewhere [King, 1965a, 1965b]. The transmitting antenna was an openended C-band waveguide which was mounted next to the plane surface of the water and aluminum. The small backscattered signal from a short vertical dipole was amplitude modulated by changing the dipole scattering characteristics at an audio rate. The scat- tering dipole used was a small switching diode in which the junction impedance was modulated with an audio oscillator through small conducting leads which had negligible effect upon the field being measured.

A number of height-gain experiments were first conducted. These have been described by King [1965b]. It was experimentally established that the open-ended waveguide transmitting antenna had radiation characteristics in the area of interest that were similar to those of a short vertical electric dipole on the surface of the ground plane. The center of the scattering dipole was maintained at a height of about $0.18 \lambda$ above the ground surface to avoid troublesome coupling effects between the diode and the surface beneath it. Height-gain corrections of the theoretical data were found to be unnecessary.

The vertical electric field intensity was measured along a line extending from the transmitting antenna across the abrupt aluminum-water boundary and perpendicular to it. A measuring system was used in which the electric field was plotted point-by-point on an $x-y$ recorder as a function of distance, $d$, from the transmitting antenna.

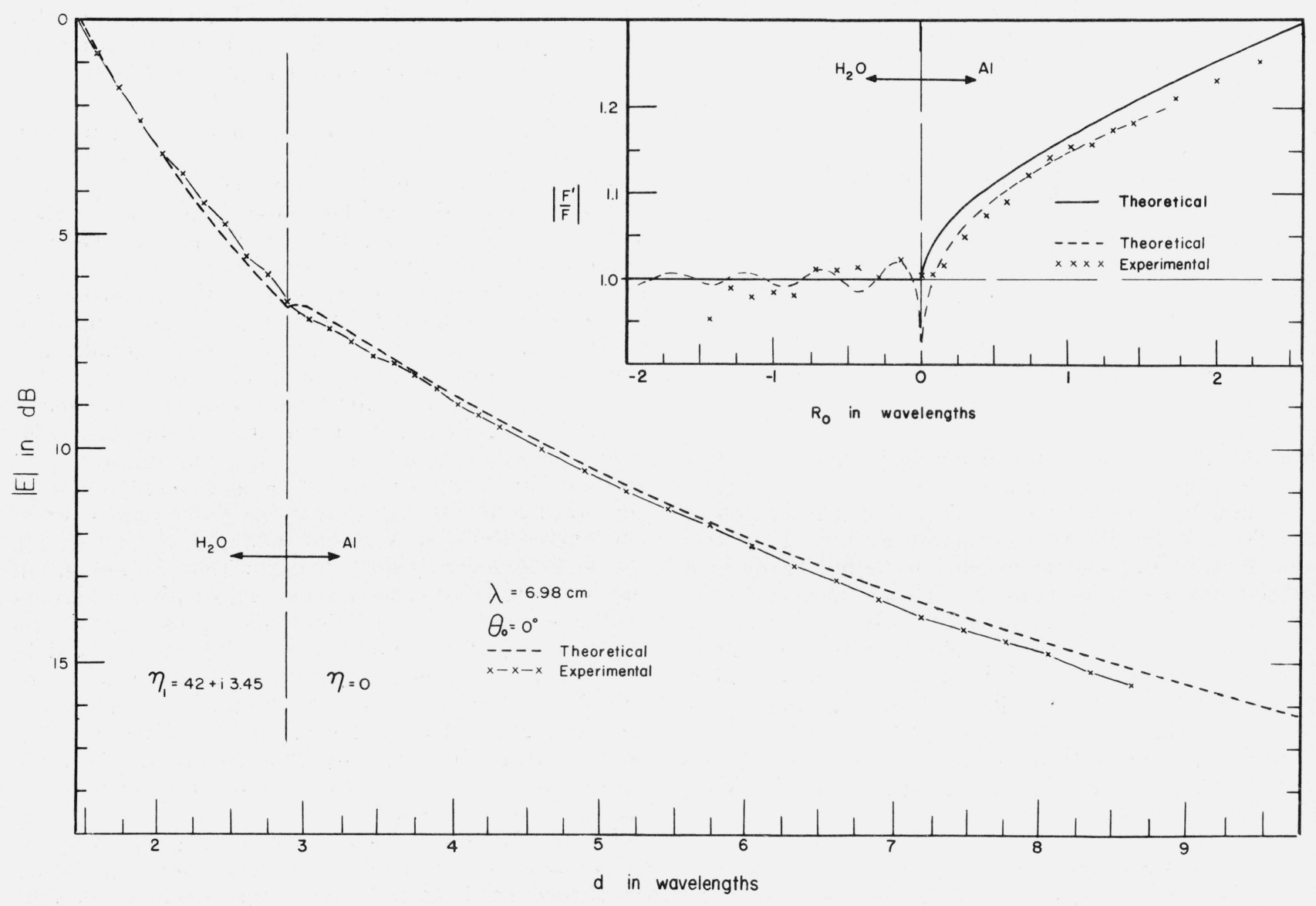

FIGURE 3. Magnitude of the vertical electric field (expressed in $d B$ relative to an arbitrary reference) versus $\mathrm{d}$ in wavelengths for perpendicular propagation across an abrupt water-to-aluminum boundary. 


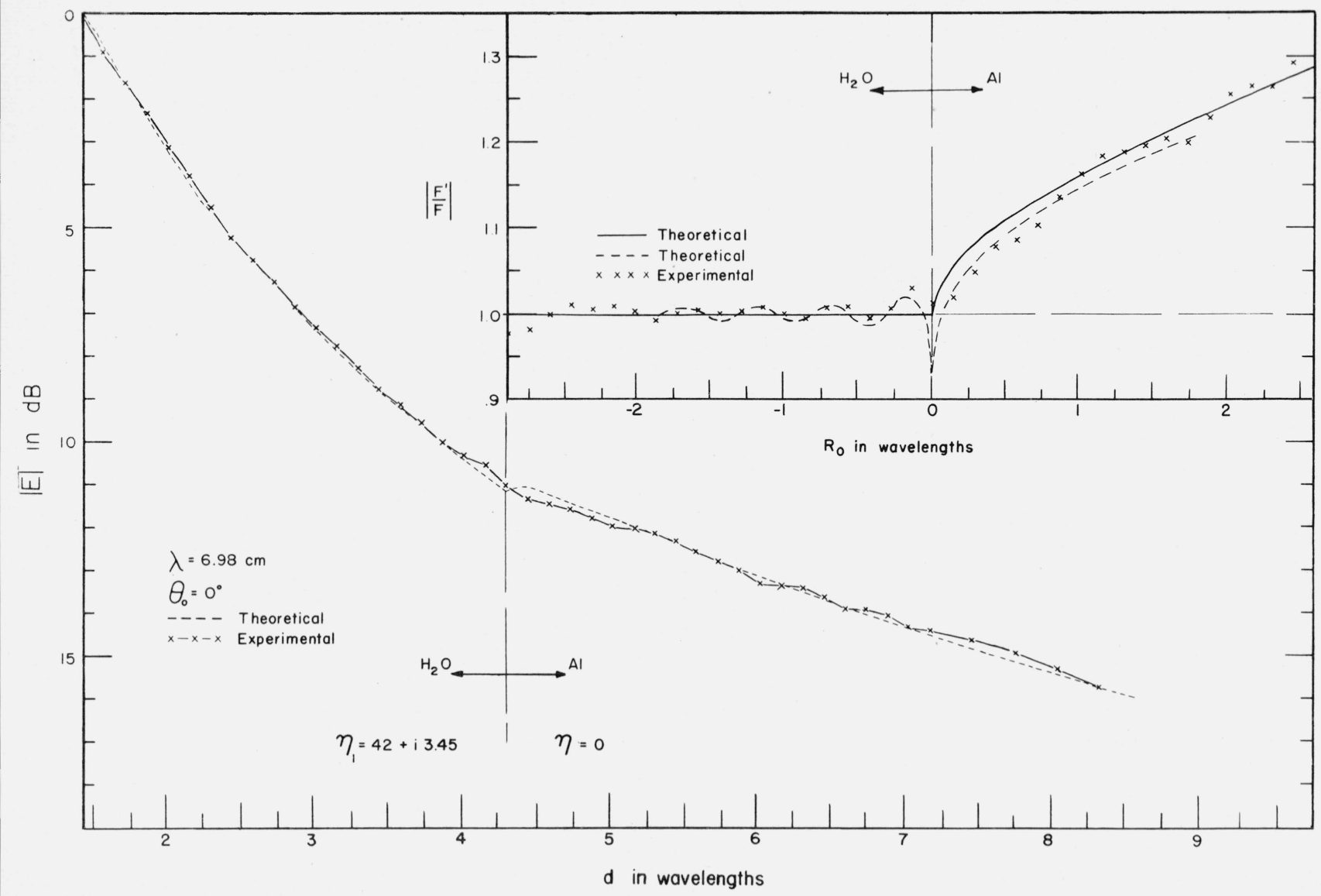

FIGURE 4. Magnitude of the vertical electric field (expressed in dB relative to an arbitrary reference) versus $\mathrm{d}$ in wavelengths for perpendicular propagation across an abrupt water-to-aluminum boundary.

The incets show the same experimental data plotted as the magnitude of the ratio $F^{\prime} / F$ versus $R_{0}$, the distance from the boundary in wavelengths. $r_{0}=4.30 \lambda(30 \mathrm{~cm})$.

\section{Results}

Figures 3 through 5 show some typical results which were obtained for propagation from water to aluminum over an abrupt boundary, and figure 6 shows similar data for propagation from aluminum to water. In all of these, both the magnitude of the relative vertical electric field (expressed in $\mathrm{dB}$ with respect to an arbitrary level) and the magnitude of the ratio, $\left|F^{\prime}\right| F \mid$, have been plotted (insets). The theoretical curves with which the experimental data for $\left|E_{Z}\right|$ have been compared were calculated using (7). These same data were plotted in the insets in addition to calculations based upon Wait's result leqs (4), or (18) and (19) in Wait, 1963, Part I] for the field near an abrupt boundary (the dashed curves). $R_{0}$ is the distance from the boundary measured in wavelengths.

It must be remembered that the impedance concept was used in both of these theoretical formulations. Therefore, calculations are not expected to be valid very near the boundary since the field changes rapidly within a ground wavelength. Furthermore, (7) is not expected to be valid within about one free space wavelength on either side of the boundary. Finally, the theory for the field near an abrupt boundary assumes $\left|\Delta Z_{m}\right| Z_{m}|=| \frac{F^{\prime}}{F}-1 \mid<<1$, and therefore it is not valid to the far right of the boundary.

In general, the results are favorable. The experimentally measured $\left|E_{z}\right|$ agrees with calculations made using (7) within $0.3 \mathrm{~dB}$ or about 3 percent. In most cases, the greatest disagreement occurs within a wavelength on either side of the boundary as expected. The experimentally determined ratio $\left|\boldsymbol{F}^{\prime} / \boldsymbol{F}\right|$ agrees reasonably well with the theory for the field near an abrupt boundary, except for $d_{1}>+1.5 \lambda$, where the assumption that $\left|\Delta Z_{m}\right| Z_{m} \mid<<1$ fails.

It is quite evident that standing waves exist in front of the boundary, and they compare reasonably well in both amplitude and phase with calculations made from (4) except right at the boundary. These standing waves are due to the interference of the incident wave and a wave which is reflected from the abrupt change of surface impedance at the boundary. The singular nature of the field very near the boundary has been partially obscured due to the nonzero 


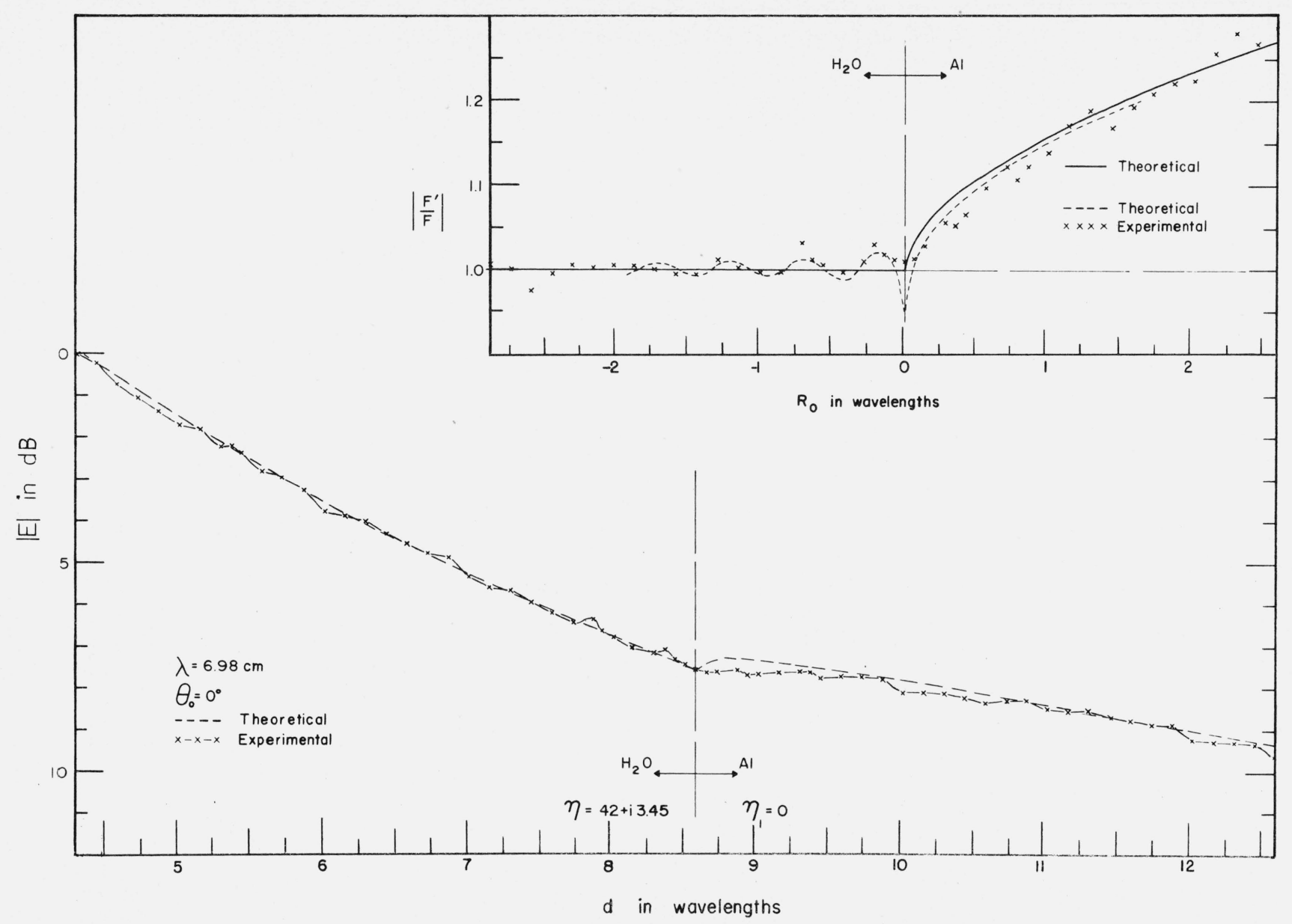

FIGURE 5. Magnitude of the vertical electric field (expressed in $d B$ relative to an arbitrary reference) versus $\mathrm{d}$ in wavelengths for perpendicular propagation across an abrupt water-to-aluminum boundary.

The insets show the same experimental data plotted as the magnitude of the ratio $F^{\prime} / F$ versus $R_{0}$, the distance from the boundary in wavelengths. $r_{0}=8.6 \lambda(60 \mathrm{~cm})$.

height of the scattering dipole and its physical size; it has also occasionally been observed in other experiments although reliable measurements were impossible.

\section{Conclusions}

Of course, the following conclusions are restricted to the parameters which were used in these model studies. However, it is appropriate to point out that the impedance contrast of an aluminum-water combination of mixed paths is quite large. Therefore, any errors present in the theory should be somewhat exaggerated in these models. An actual impedance contrast at VLF frequencies will usually be less, making the integrals in these calculations smaller, resulting in smaller errors in most cases.

The results indicate that calculations based upon (7) are accurate within 2 to 3 percent. The largest errors are likely to occur within a free space wavelength on either side of the abrupt boundary. Calcu- lations based upon (4) have been shown to be accurate up to 1 wavelength behind the boundary where $\left|\Delta Z_{m}\right| Z_{m} \mid$ becomes comparable to unity. For greater values of $d_{1},(7)$ is recommended. It has been shown to be accurate when the boundary is located as close as 3 wavelengths to the transmitting antenna, and there is good reason to believe it is accurate when the boundary is even closer.

The research reported here was supported by Air Force Cambridge Research Laboratories, Bedford, Mass., PRO-62-201, under subcontract by the Central Radio Propagation Laboratory, National Bureau of Standards, Boulder, Colo., the University of Colorado Council on Research, and the National Science Foundation under contract No. GK-85.

The authors thank J. R. Wait for his many helpful suggestions, D. K. Hadley who conducted many of the experiments and J. I. Gilmer who plotted the experimental and theoretical data. 


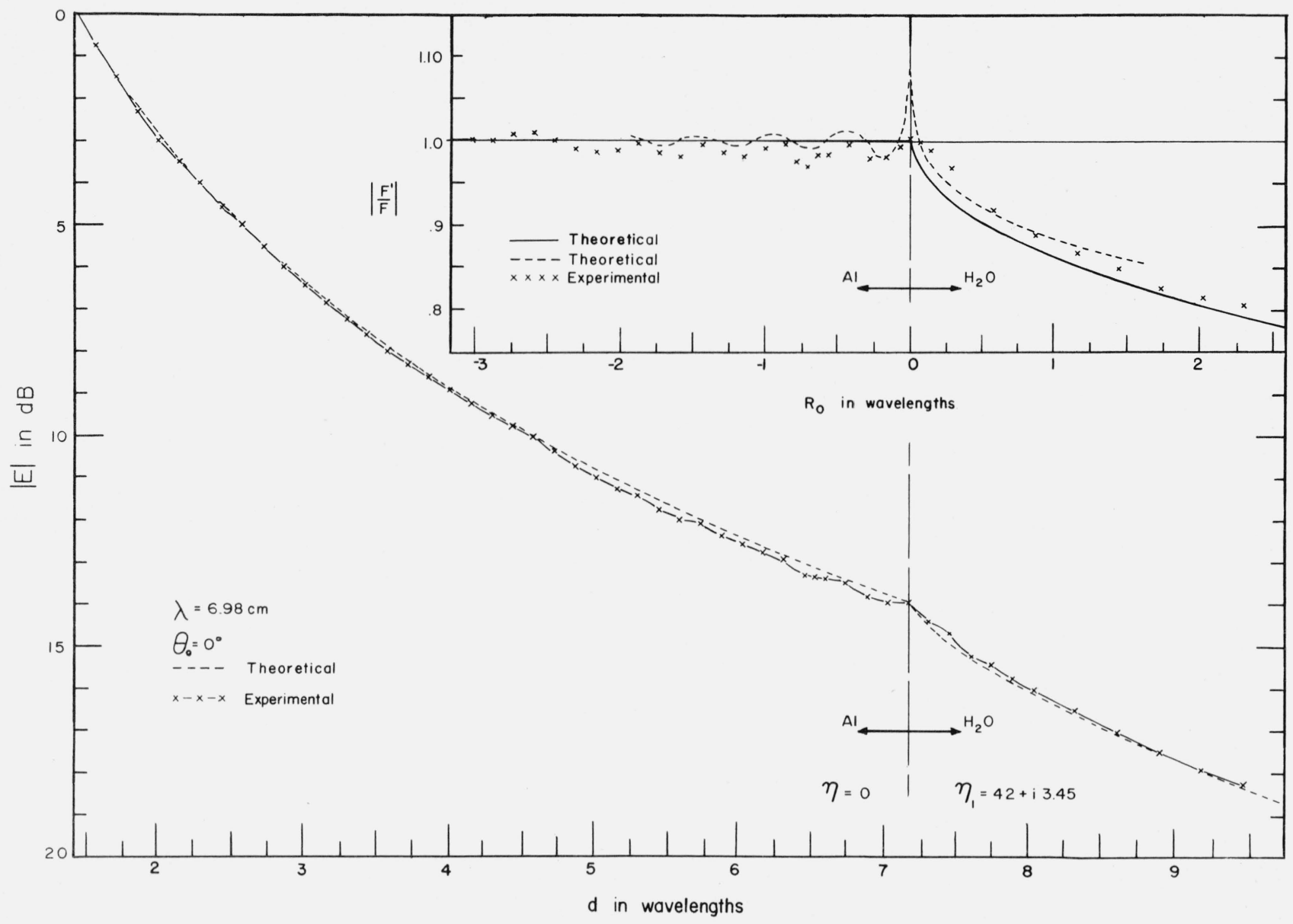

FigURE 6. Magnitude of the vertical electric field (expressed in $d B$ relative to an arbitrary reference) versus $\mathrm{d}$ in wavelengths for perpendicular propagation across an abrupt aluminum-to-water boundary.

The insets show the same experimental data plotted as the magnitude of the ratio $F^{\prime} / F$ versus $R_{0}$, the distance from the boundary in wavelengths. $r_{0}=7.15 \lambda(50 \mathrm{~cm})$.

\section{References}

Furutsu, K. (1955), Propagation of electromagnetic waves over a spherical earth across boundaries separating different media, J. Radio Res. Lab. (Tokyo) 2, 1-49 and 345-398.

Furutsu, K. (1956), The calculation of field strength over mixed paths on a spherical earth, J. Radio Res. Lab. (Tokyo) 3, No. 14, 391407.

Furutsu, K. (1963), On the theory of radio wave propagation over an inhomogeneous earth, J. Res. NBS 67D (Radio Prop.) No. 1, 39-62. This paper also gives other references by the author.

King, R. J. (Mar. 1965a), An amplitude and phase measuring system using a small modulated scatterer, Microwave J. 8, No. 3, 51 .

King, R. J. (1965b), Analytic and experimental studies of propagation of electromagnetic surface waves across mixed paths, Ph. D. thesis, University of Colorado, Boulder, Colo.

Maley, S. W., and H. Ottesen (1964), An experimental study of mixed-path groundwave propagation, Radio Sci. J. Res. NBS 68D, No. 8, 915-918.

Wait, J. R. (July 1956), Mixed-path groundwave propagation; I. Short distances, J. Res. NBS 57, No. 1, 1-15, RP2687.

Wait, J. R.(1961), On the theory of mixed-path groundwave propagation on a spherical earth. J. Res. NBS 65D (Radio Prop.), No. 4, 401-410.
Wait, J. R. (1962), Electromagnetic Waves, ed. Rudolph E. Langer, ch. on Propagation of Electromagnetic waves Along the Earth's Surface, pp. 243-298 (University of ${ }^{\top} \mathrm{W}$ isconsin Press).

Wait, J. R. (Nov.-Dec. 1963), Oblique propagation of groundwaves across a coastline. Part I, J. Res. NBS 67D (Radio Prop.) No. 6, 617-624.

Wait, J. R. (1964a), Advances in Radio Research, ed. J. A. Saxton, 1, ch. on Electromagnetic Surface Waves, pp. 157-217 (Academic Press, New York, N.Y.).

Wait, J. R. (1964b), Oblique Propagation of groundwaves across a coastline. Part III, Radio Sci. J. Res. NBS 68D, No. 3, 291-295.

Wait, J. R., and J. Householder (July 1957), Mixed-path groundwave propagation: 2. Larger distances, J. Res. NBS 59, No. 1, 19-26, RP2770.

Wait, J. R., and C. M. Jackson (Nov.-Dec. 1963), Oblique propagation of groundwaves across a coastline. Part II, J. Res. NBS 67D (Radio Prop.), No. 6, 625-630.

Wait, J. R., and K. P. Spies (1964), Propagation of radio waves past a coastline with a gradual change of surface impedance, IEEE Trans. Ant. Prop. AP-12, No. 5, 570-575.

(Paper 69D10-571) 


\section{Reviews and selected abstracts in radio science*}

\begin{abstract}
Practical low-loss waveguide for long-distance telecommunications, H. E. M. Barlow, H. G. Effemey, and S. H. Taheri, Electronics Letters 1, No. 1, 14-15 (Mar. 1965).

A description is given of a form of waveguide circuit operated over a frequency range of $30-40 \mathrm{Gc} / \mathrm{s}$ and incorporating a number of bends having only a 4 in radius of curvature; it is demonstrated to transmit successfully amplitude-modulated television pictures and sound side by side with short microwave pulses. Details of circuit arrangements and modulation technique are given.
\end{abstract}

Surface-wave probe for measuring electron densities in a gaseous plasma, P. N. Robson and R. D. Stewart, Electronics Letters 1, No. 1, 13-14 (Mar. 1965).

A dielectric rod enveloped in a gaseous plasma can act as a supporting structure for electromagnetic surface waves. The dielectric constant of the plasma and hence the electron number density in the vicinity of the rod may be determined from measurements of the phase-change coefficient of the $\mathrm{EH}_{11}$ mode supported by the plasmaclad rod. Measurements of radial density distribution have been carried out in an unmagnetized argon afterglow plasma at $35 \mathrm{Gc} / \mathrm{s}$ and compared with double-probe measurements.

Guided waves on a dielectric-coated cylinder immersed in a plasma, S. R. Seshadri, Electronics Letters 1, No. 1, 19 (Mar. 1965). The dominant circularly symmetric TM surface-wave mode on a Goubau wire immersed in an isotropic plasma is shown to have a low-frequency cutoff, in contrast to its free-space counterpart, for which the propagation extends down to zero frequency.

Dielectric measurement of high-loss liquids in rectangular waveguides, J. A. Staniforth, R. G. Bennett, and J. H. Calderwood, Electronics Letters 1, No. 1, 4 (Mar. 1965).

The note extends for use in rectangular waveguides a method of measuring the dielectric constants of high-loss liquids previously described by Bennett and Calderwood.

Effects of electron-density distribution on the backscattering from an anisotropic plasma cylinder, C. Yeh and W. V. T. Rusch, Electronics Letters 1, No. 1, 25 (Mar. 1965).

The backscattering cross-section of a radially inhomogeneous plasma cylinder with an impressed axial static magnetic field is obtained for an incident $H$ wave. The electron-density distribution is assumed to have a parabolic profile. Significant variation of the backscattering cross-section as a function of the density profile is found.

Modes in spherical-mirror resonators, W. A. Specht, Jr., J. Appl. Phys. 36, No. 4, 1306-1313 (Apr. 1965).

This work is the examination of a cavity mode approach to the mode structure of a laser. Solutions of the vector wave equation for electromagnetic fields in and between perfectly conducting oblate spheroidal cavities are examined for the case of wavelengths much less than cavity dimensions. These solutions are the field modes in Fabry-Perot type resonators with equal-radius concave spherical mirrors, or with concave-convex spherical mirrors, when the parameters of the oblate spheroids are chosen so that the radii of curvature and spacing on the axis of rotation match those of the resonator mirrors. Expressions for the transverse and longitudinal mode structures are derived. The eigenvalue equations are written, and are solved for the case of the two lowest-order modes.

Fields produced by a current source in a partly ionized gas, B. Samuel Tanenbaum, J. Appl. Phys. 36, No. 4, 1288-1290 (Apr. 1965).

A technique is developed for treating each of the waves generated by an arbitrary current source in a partly ionized gas with no steady magnetic field. Starting with Maxwell's equations and three sets of coupled hydrodynamic equations (for the electrons, ions, and neutral molecules), it is shown that one can obtain a separate inhomogeneous wave equation for each type of wave in the plasma, with the source term for the transverse and longitudinal waves dependent, respectively, on the curl and divergence of the current source.

Measurement of $\mathrm{H}_{01}$ mode loss at $Q$ band in helix waveguide, J. A. Berry, Marconi Rev. 28, No. 156, 22-26 (1st quarter, 1965).

This article deals with one method of measuring the $\mathrm{H}_{01}$ circular mode loss in a circular guide in the frequency range $26.5-40 \mathrm{Gc} / \mathrm{s}$. The cylindrical wall of the circular guide, of internal diameter 2 in., was formed from enameled copper wire wound in a helical fashion. From this, the name "helix waveguide" is coined. The measured loss varied from approximately $4 \mathrm{dBs}$ per mile at $40 \mathrm{Gc} / \mathrm{s}$ to 6.5 $\mathrm{dBs} / \mathrm{mile}$ at $26.5 \mathrm{Gc} / \mathrm{s}$.

Coupling between modes in helix waveguide, D. J. Bowe and R. A. Waldron, Marconi Rev. 28, No. 156, 65-88 (1st quarter, 1965).

Results are given of computations of coupling coefficients between the $\mathrm{H}_{01}$ mode and various other modes in helix waveguide, for coupling due to the following imperfections: changes of radius, offsets of the axes of joined lengths of guide, sharp bends of the axis, and curved bends. The design of curved bends is discussed, and results are given for the effects of random small curvature over long distances, using a new theory for integrating the effects over appreciable distances of continuous coupling. An unexpected result that was obtained is that the coupling coefficient for a small-angle bend is the same for a sharp bend as for a curved bend; the coupling depends only on the angle of bend, not on the way in which the bend is made.

The $\mathbf{H}_{01}$ mode and the helix waveguide, M. H. Cufflin, Marconi Rev. 28, No. 156, 1-21 (1st quarter, 1965).

This article deals with the experimental work on the low loss $\mathrm{H}_{01}$ mode in circular waveguide carried out in the research Division of the Marconi Company during the last four years. The work began as an investigation into the transmission problems involved in long distance communication by waveguide using millimetre wavelengths. Later work was carried out at longer wavelengths in the $3 \mathrm{~cm}$. and $6 \mathrm{~cm}$. bands to test the suitability of the techniques to achieve very low losses for high-power feeds for radars or low noise receiving aerials. Results on an X-band radar are included.

Normal modes of helix waveguide, R. A. Waldron and D. J. Bowe, Marconi Rev. 28, No. 156, 29-64 (1st quarter, 1965).

An outline is given of the derivation, in helical co-ordinates, of the characteristic equation for helix waveguide. A selection is presented of results of computations of the (complex) phase constant, for a number of values of the normalized guide radius and of the properties of the materials.

Statistics of the coupling into unwanted modes in a long multi-mode transmission system, B. Westcott, Marconi Rev. 28, No. 156, 89-101 (1st quarter, 1965).

The effects of discontinuous defects, occurring at the junctions between sections of helix waveguide in a long-distance communications system, are considered statistically. The defects are divided into two classes according as the unwanted modes coupled into have large or small attenuation constants. Expressions are derived for the average power coupled into the unwanted modes. At certain frequencies the power in modes having low attenuation constants may build up to unduly large amounts, thus increasing the risk of distortion of the wanted mode. It is shown that the probability of this happening can be minimized by randomising the section lengths with rectangular or normal distributions having standard deviations of a few centimetres. 
The following publication is available from American Elsevier Publishing Company, 52 Vanderbilt Avenue, New York, N.Y., 10017. The book contains review papers presented at Commission III on Ionospheric Radio During the XIVth General Assembly of URSI, Tokyo, September, 1963.

Progress in Radio Science 1960-1963, Volume III: The Ionosphere, edited by Geoffrey M. Brown, Prefạce, v.

At the XIVth General Assembly of the International Scientific Radio Union (URSI) held in Tokyo over the period September 9-20, Commission III, on the Ionosphere, held nine scientific sessions, some of which were in collaboration with Commission IV, on the Magnetosphere. Each session was devoted to a consideration of a particular subject, and was introduced by the presentation of an extended review paper prepared by an invited speaker, prior to shorter contributions and a general discussion.

The subjects discussed included the distribution of electrons throughout the whole ionosphere from the $D$-region, through the topside ionosphere, to the magnetosphere and interplanetary space; interrelations between the ionosphere and geomagnetism; ionospheric studies during the IQSY; ionizing radiation and the production of the ionosphere; and the constitution of the atmosphere, including a consideration of the history of the earth's atmosphere from its primordial state.

The full text of each of the introductory survey papers is given in this volume, together with a summary of progress in ionospheric physics over the period 1960-63 prepared by the Chairman of URSI Commission III, Mr. J. A. Ratcliffe. This paper outlines some of the main advances which have been published over the triennium since the last General Assembly and also summarizes the chief points which emerged from the discussions at this Assembly.

Advances in Ionospheric Physics 1960-1963, J. A. Ratcliffe, Radio Research Station, Slough, Bucks. (England), pp. 1-13.

Some of the more important advances in knowledge of the ionosphere and upper atmosphere which have occurred in the triennium 1960-63 are summarized. The review is based on the National Reports presented by the member countries to the XIVth General Assembly of URSI in 1963, together with the discussions which took place at the scientific sessions of Commission III during this assembly.

The Distribution of Electrons in the Lower and Middle Ionosphere, R. W. Knecht, National Bureau of Standards, Boulder, Colo. (U.S.A.), pp. 14-45.

A review of current knowledge of the distribution of electrons in the $D-, E$-, and lower $F$-regions of the ionosphere is presented. Particular emphasis is put on advances made during the triennium 1960-1963. Noteworthy is the increase in the amount of data available on the electron density profile in the $D$-region from groundbased and rocket experiments. Also, the shape of the height profile of electrons in the $E$ - and lower $F$-region has been significantly refined through the use of improved vertical sounders and by an increasing number of in situ observations. Information has been obtained by means of rocket experimentation on the nature of the ionization structures responsible for certain types of sporadic- $E$.

A Survey of Topside Sounding of the Ionosphere, J. H. Chapman, Defence Res. Telecommun. Estab., Defence Research Board, Ottawa (Canada), pp. 46-64.

Topside sounding experiments have been conducted using rocketborne fixed-frequency sounders in June and October 1961, and a sweep-frequency topside sounder in the satellite 1962 Beta Alpha One (Alouette) launched 29 September 1962. This paper reviews the techniques of topside sounding, and some of the more significant results obtained from these experiments.

Some Results of U.S.S.R. Experiments in the Ionosphere and Interplanetary Space, K. I. Gringauz, Radio Tech. Inst., Academy of Sciences, Moscow (U.S.S.R.), pp. 65-75.

A review is given of the results of some experiments made in 1961-62 which are a part of the Soviet programme of upper atmospheric and interplanetary space investigations. These include rocket measurements of electron densities and electric fields in the ionosphere, ion density and composition changes in the upper ionosphere determined by the satellite Cosmos 2, and plasma measurements in the magnetosphere and interplanetary space made in the space probe Mars 1.
Whistler Measurements of the Equatorial Profile of Magnetospheric Electron Density, D. L. Carpenter, Radioscience Laboratory, Stanford, Calif. (U.S.A.), pp 76-91.

A whistler method of deducing the equatorial profile of electron density is described. Results of several investigations are compared, and it is found that there is excellent agreement on the shape of the profile. Its average behavior in the range of $2 R_{E}$ to $5 R_{E}$ geocentric distance is estimated to fall between about $N \propto R^{-3}$ and $N \propto R^{-4}$. Several roughly comparable estimates of numerical values are found to be within a factor of about 2 . It is found that the results from whistlers are generally consistent with $5000-\mathrm{km}$ values of electron density obtained by incoherent scatter measurements at the geomagnetic equator.

Whistler evidence of a knee in the equatorial profile is presented. The knee is characterized by a region in which the profile drops rapidly from a relatively normal level to a level that may be depressed by a factor of 6 or more. The knee is believed to be a persistent phenomenon, which moves inward with increasing magnetic activity. Measurements made by the Lunik I and Lunik II probes are found to be consistent with whisper predictions of a knee in the equatorial profile.

Some of the recently obtained results on temporal variations in magnetospheric density are discussed.

Geomagnetism and the Ionosphere, C. O. Hines, University of Chicago (U.S.A.), pp. 92-120.

Interactions between the geomagnetic field and the ionosphere are reviewed. The large-scale current systems of quiet days and of magnetic storms receive the greatest attention with emphasis being placed on the points of uncertainty in the former case and points of agreement in the latter. The larger-scale rotational system and smaller-scale irregularities and wave interactions are surveyed as well, but only briefly. The whole discussion is set against a background summary of the hydromagnetic approximation, which permits an economy of thought that is useful in developing and retaining a picture of events adequate for many purposes.

Some Comments on the Ionosphere and Geomagnetism, E. H. Vestine, The RAND Corporation, Santa Monica, Calif.(U.S.A.), pp. 121-148.

The broad-scale circulation and dynamics of the magnetosphere are discussed in relation to some problems of the ionosphere. The importance of electric fields and charge separations in the production of various localized features of the magnetosphere seems as sured. Many of the features discussed may be instructively interpreted in terms of the Chapman and Ferraro theory.

Ionospheric Studies During the IQSY 1964-1965, W. J. G. Beynon, University of Wales, Aberystwyth (U.K.), pp. 149-166.

Some of the more important results obtained during and since the IGY are reviewed and discussed in the light of the programme of observations for the IQSY.

Ionizing Radiation and Constitution of the Atmosphere, Herbert Friedman, E. O. Hulburt Center for Space Research, U.S. Naval Res. Lab., Washington, D.C., pp. 167-173.

Progress in determining the emission line spectrum of solar ionizing radiation under quiet and disturbed conditions is summarized, and applied to considerations of the formation of the ionosphere. The role of a corpuscular contribution to ionization is also briefly discussed.

The History of Growth of Oxygen in the Earth's Atmosphere, L. V. Berkner and L. C. Marshall, Southwest Center for Advanced Studies, Dallas, Texas (U.S.A.) pp. 174-196.

There is considerable evidence that upon its agglomeration the earth was without a primordial atmosphere, so that the subsequent development of an atmosphere must be ascribed to secondary sources. This paper discusses the history of this development from the primitive origin of oxygen by photochemical processes to the buildup of the present oxygen level by photosynthesis. The study leads to new implications with regard to the physical and biological history of the earth. 


\section{Selected Abstracts of Publications of the Staff of NBS}

Radio refractometry and its potential for humidity studies, R. E. McGavin and M. J. Vetter, International Symposium on Humidity and Moisture, Washington, D.C., 1963. Humidity and Moisture 2. Applications, pp. 553-560 (Reinhold Publ. Corp., New York, N.Y., 1965).

Radio refractometers have been in use for the past decade. Their primary use has been in the investigation of the refractive index structure as applied to radio-wave propagation. There has been considerable improvement in these devices in recent years and the present status of radio refractometry indicates broader application of these techniques. Since the radio refractive index is a function of pressure, temperature and humidity, these devices can be converted to hygrometers when suitable compensation for temperature and pressure is applied. This technique has been used successfully by a number of investigators. The results of some of these investigations are reviewed.

Free electron balance during polar cap absorption events, L. R. Megill and J. B. Hasted, Planetary Space Sci. 13, No. 4, 339-343 (Apr. 1965).

Critical examination is made of a proposal that in polar cap absorption events electrons are detached from $\mathrm{O}_{2}^{-}$by $\mathrm{O}_{2}\left({ }^{1} \Delta_{g}\right)$. A rate of $10^{-9} \mathrm{~cm}^{3} \mathrm{~s}^{-1}$ is suggested on the basis of microwave studies of the oxygen afterglow; the temporal behaviour during sunlight hours is explained without the need for invoking another negative ion. It is suggested that the requirement for a large deactivation rate by ground state $\mathrm{O}_{2}$, which is in contradiction with experiment, is not required if moderate concentrations of metastable molecules are postulated at night.

Longitude distribution of proton flares, Constance S. Warwick, Astrophys. J. 141, No. 2, 500-504 (Feb. 1965).

Energetic protons that cause polar-cap absorption (PCA) come from flares that cluster in certain preferred heliographic longitudes. This clumping of great flares at locations that are fixed in a rigidly rotating system is distinct from the recurrence or revival of individual long-lived active regions. The clustering of PCA flares is related to the clustering of optical solar activity found by Trotter and Billings in cycle 19, but the grouping of PCA flares is much sharper. The results of sunspot studies by Losh and by Vitinskii are shown to be consistent with each other when both are presented in the same form.

\section{NBS Publications}

Ionospheric radio propagation, K. L. Davies, NBS Mono. 80 (Apr. 1965), $\$ 2.75$.

Examination of liquefied petroleum gas liquid-measuring devices, M. W. Jensen, NBS Handb. 99 (Apr. 23, 1965), 35 cents.

Systems engineering in ceramics. Proceedings of a symposium April 19, 1964, NBS Misc. Publ. 267 (May 1, 1965), \$2.00.

Steel medicine cabinets, NBS CS267-65 (Mar. 1, 1965), 10 cents.

Hide trim pattern for domestic cattlehides, NBS CS268-65 (Apr. 4, 1965) 10 cents.

Research on crystal growth and characterization at the National Bureau of Standards July to December 1964, ed. H. F. McMurdie, NBS Tech. Note 260 (May 8, 1965), 50 cents.

Studies of solar flare effects and other ionospheric disturbances with a high frequency Doppler technique, V. Agy, D. M. Baker, and R. M. Jones, NBS Tech. Note 306, (Apr. 28, 1965), 75 cents.

Phase and amplitude diversity in over-water transmissions at two microwave frequencies, H. B. Janes, A. W. Kirkpatrick, D. M. Waters, and D. Smith, NBS Tech. Note 307 (Apr. 12, 1965), 40 cents.

Data reduction for stable auroral red arcs observed at Rapid City, South Dakota, J. E. Cruz, R. Davies, L. K. Droppleman, E. Marovich, L. R. Megill, M. H. Rees, L. Reisbeck, and F. E. Roach, NBS Tech. Note 308 (May 3, 1965), 50 cents.

A bibliography of experimental saturation properties of the cryogenic fluids, N. A. Olien and L. A. Hall, NBS Tech. Note 309 (Apr. 9, 1965), 60 cents.

Attenuation of the ground wave of a low frequency electromagnetic pulse, J. C. Morgenstern and J. R. Johler, NBS Tech. Note 310 (Apr. 1965), 30 cents.
A line-formula notation system for coordination compounds, P. M. McDonnell and R. F. Pasternack, J. Chem. Documentation 5, No. 1, 56-60 (Feb. 1965).

Decyclization of cyclohexene, 4-methylcyclohexene, and 4-vinylcyclohexene in a single-pulse shock tube, W. Tsang, J. Chem. Phys. 42, No. 5, 1805-1809 (Mar. 1, 1965).

Direct and inert-gas-sensitized radiolysis and photolysis of methane in the solid phase, P. Ausloos, R. E. Rebbert, and S. G. Lias, J. Chem. Phys. 42, No. 2, 540-548 (Jan. 15, 1965).

Effect of electrical fields and density in the radiolysis of ethane, H. H. Carmichael, R. Gorden, Jr., and P. Ausloos, J. Chem. Phys. 42, No. 1, 343-351 (Jan. 1, 1965).

Equilibrium pressures of oxygen over $\mathrm{MnO}_{2}-\mathrm{Mn}_{2} \mathrm{O}_{3}$ at various temperatures, E. M. Otto, J. Electrochem. Soc. 112, No. 4, 367-370 (Apr. 1965).

Hydrogen atom addition to olefins: Relative rates at the two carbon positions and derived heats of formation of several alkyl radicals, R. D. Kelley, R. Klein, and M. D. Scheer, J. Phys. Chem. 69, No. 3, 905-908 (Mar. 1965).

Isotopic mixing in $\mathrm{CO}$ chemisorbed on tungsten. A kinetic study, T. E. Madey, J. T. Yates, Jr., and R. C. Stern, J. Chem. Phys. 42, No. 1, 1372-1378 (Feb. 15, 1965).

Some new co-ordination compounds of copper (II) chloride, C. Reimann and G. Gordon, Nature 205, No. 4974, 902-903 (Feb. $27,1965)$.

Standardization of analytical data obtained with silver-silver chloride electrodes in methanol-water solvents, M. Paabo, R. G. Bates, and R. A. Robinson, Anal. Chem. 37, 462-464 (1965).

Watermarks for papermaking by electroforming, J. P. Young, Tappi 48, No. 1, 36A-37A (Jan. 1965); Metal Finishing 63, No. 4, 79-82 (Apr. 1965).

Latensification in radiographic emulsions, W. L. McLaughlin, J. Photo. Sci. 13, 1-11 (1965).

On the information in a microphotograph, C. S. McCamy, Appl. Opt. 4, No. 4, 405-411 (Apr. 1965).

Results of National Bureau of Standards corrosion investigation in disturbed and undisturbed soils, M. Romanoff, Proc. Appalachian Underground Short Course, Tech. Bull. 72, pp. 566-589 (West Virginia Univ., Morgantown, West Va., 1965).

Statistical design of experiments, W. J. Youden, Handbook on Metals Engineering Design, American Society of Mechanical Engineers, ch. 3, Pt. 5, pp. 583-585 (McGraw-Hill Publ. Corp., New York, N.Y., 1965).

Technique for determining spin-lattice relaxation times, G. A Candela and R. E. Mundy, Rev. Sci. Instr. 36, No. 3, 338-342 (Mar. 1965).

Two cases of stress corrosion cracking in copper tubing, H. L. Logan and G. M. Uginsky, Materials Protection 4, No. 3, 79-80 (May 1965).

A uniqueness theorem for entire functions, R. F. DeMar, Proc. Am. Math. Soc. 16, No. 1, 69-71 (Feb. 1965).

Nomograms for computing real, imaginary, and absolute values of vector ratios, H. S. Bowman, Acoust. Soc. Am. 37, No. 4, 751 (Apr. 1965).

On a theorem concerning existence of interpolating functions, R. F. DeMar, Trans. Am. Math. Soc. 114, No. 1, 23-29 (Jan. 1965).

A study of errors in the measurement of microscopic spheres, C. P. Saylor, Appl. Opt. 4, No. 4, 477-486 (Apr. 1965).

Definition of "ampere" and "magnetic constant," C. H. Page, Proc. IEEE 53, No. 1, 100-101 (Jan. 1965).

Electromagnetic measurement, H. W. Lance, SPACE/AERONAUTICS Res. Develop. Handb. 42, No. 4, 199-202 (19641965).

A proper accounting of conformation of a polymer near a surface, E. A. DiMarzio, J. Chem. Phys. 42, No. 6, 2101-2106 (Mar. 1965).

A test of analytical expressions for the thermal emissivity of shallow cylindrical cavities, F. J. Kelly and D. G. Moore, Appl. Opt. 4, No. 1, 31-40 (Jan. 1965).

A universal color language, K. L. Kelly, Color Eng. 3, No. 2, 16-21 (Mar--Apr. 1965).

Characteristics of the "synchrotron light" from the NBS-180 $\mathrm{MeV}$ machine, K. Codling and R. P. Madden, J. Appl. Phys. 36, No. 2, 380-387 (Feb. 1965).

Dependence of the superconducting transition temperature on carrier concentration in semiconducting $\mathrm{SrTiO}_{3}$, J. F. Schooley, W. R. Hosler, E. Ambler, J. H. Becker, M. L. Cohen, and C. S. Koonce, Phys. Rev. Letters 14, No. 9, 305-307 (Mar. 1, 1965). 
Direct observation of the optical anisotropy of the holmium nucleus, E. Ambler, E. G. Fuller, and H. Marshak, Phys. Rev. 138, No. 1B, B117-B126 (Apr. 12, 1965).

Growth layers on ammonium dihydrogen phosphate, J. L. Torgesen and R. W. Jackson, Science 148, No. 3672, 952-954 (May 14, 1965).

High silica glass, quartz, and vitreous silica, J. S. Laufer, J. Opt. Soc. Am. 55, No. 4, 458-460 (Apr. 1965).

Influence of paramagnetic resonance on the static susceptibility. Spin-lattice relaxation time of cupric sulfate pentahydrate, G. A. Candela, J. Chem. Phys. 42, No. 1, 113-117 (Jan. 1, 1965).

Interference patterns in reverberant sound fields. II, R. V. Waterhouse and R. K. Cook, J. Acoust. Soc. Am. 3 7, No. 3, 424-428 (Mar. 1965).

Line profiles in the far-uv absorption spectra of the rare gases, $\mathrm{U}$. Fano and J. W. Cooper, Phys. Rev. 137, No. 5A, A1364-A1379 (Mar. 1, 1965).

Low temperature magnetic transitions in some rare-earth trichlorides, J. C. Eisenstein, R. P. Hudson, and B. W. Mangum, Phys. Rev. 137, No. 6A, Al886-A1895 (Mar. 15, 1965).

Multichannel resonances in the forward scattering of electrons by helium, G. E. Chamberlain, Phys. Rev. Letters 14, No. 15, 581582 (Apr. 12, 1965).

Nonlinear ambipolar diffusion of an isothermal plasma across a magnetic field, E. R. Mosburg, Jr., and K. B. Persson, Phys. Fluids 7, No. 11, 1829-1833 (Nov. 1964).

Polymorphism in hydrogen iodide, F. A. Mauer, C. J. Keffer, R. B. Reeves, and D. W. Robinson, J. Chem. Phys. 42, No. 4, 14651466 (Feb. 15, 1965).

Stability of traveling waves in lasers, J. A. White, Phys. Rev. 137, No. 6A, Al651-A1654 (Mar. 15, 1965).

Temperature and mean free path dependence of the Ginzburg Landau parameter, S. Gygax and R. H. Kropschot, Phys. Letters 12, No. 1, 7-9 (Sept. 1, 1964).
The measurement of water vapor boundary layers in biological systems with a radio refractometer, D. M. Gates (Proc. 1963 Intern. Symp. Humidity and Moisture, Washington, D.C.), Book, Humidity and Moisture. Measurement and Control in Science and Industry. Vol. 2. Applications, Sec. I, Paper 5, pp. 33-38 (Reinhold Publ. Corp., New York, N.Y., 1965).

The use of terahertz photobeats for precise velocity-of-light measurement, Z. Bay and H. S. Boyne, Proc. Enrico Fermi International School of Physics: Course No. XXXI Quantum Electronics and Coherent Light 1963, 352-371 (1964).

Two-electron excitation states in helium, R. P. Madden and K. Codling, Astrophys. J. 141, No. 2, 362-375 (Feb. 15, 1965).

Cavity resonances for spherical earth with a concentric anisotropic shell, J. R. W ait, J. Atmos. Terres. Phys. 27, 81-89 (1965).

Potential use of passive probing of atmospheric structure by thermal emissions at radio frequencies, B. R. Bean, E. R. Westwater, and R. L. Abbott (Proc. 1963 Intern. Symp. Humidity and Moisture, Washington, D.C.), Book, Humidity and Moisture. Measurement and Control in Science and Industry. Vol. 2, Applications, Sec. VII, Paper 71, pp. 595-608 (Reinhold Publ. Corp., New York, N.Y., 1965).

The use of the radio refractometer to measure water vapor turbulence, B. R. Bean and R. E. McGavin (Proc. 1963 Intern. Symp. Humidity and Moisture, Washington, D.C.), Book, Humidity and Moisture. Measurement and Control in Science and Industry. Vol. 2, Applications, Sec. VII, Paper 67, pp. 561-568 (Reinhold Publ. Corp., New York, N.Y., 1965).

* Publications for which a price is indicated are available by purchase from the Superintendent of Documents, U.S. Government Printing Office, Washington, D.C., 20402 (foreign postage, one-fourth additional). Reprints from outside journals and the NBS Journal of Research may often be obtained directly from the authors.

\section{CALL FOR PAPERS}

Technical Papers are solicited for the 1966 IEEE Regional Six Annual Conference which will take place April 26-28, 1966 in Tucson, Arizona, at the Pioneer Hotel. The conference will feature a truly exceptional program of technical papers and invited speakers. Among the speakers will be Dr. W. G. Shepherd, University of Minnesota; Dean W. L. Everitt of the University of Illinois; and Dr. Richard Bellman of the Rand Corporation. Among the Session Chairmen will be Dr. N. Abramson, Harvard University; Dr. J. A. Aseltine, Space Technology Laboratories; Dr. A. V. Balakrishnan, University of California at Los Angeles; Dr. N. Hilberry, University of Arizona; Mr. E. W. Kimbark, Bonneville Power Administration; Dr. G. A. Korn, University of Arizona; Dr. G. Leitmann, University of California; Dr. I. A. Lesk, Motorola Semiconductor Products Division; Dr. A. Papoulis, Polytechnic Institute of Brooklyn; Dr. D. O. Pederson, University of California; Dr. M. E. Van Valkenburg, University of Illinois; Dr. J. R. Wait, National Bureau of Standards.

List of Topics

The papers may be of a research or a tutorial nature. They should not have been previously published or presented, and should describe contributions in the following or related fields:
1. Communication Systems
2. Medical Electronics
3. Automatic Control
4. Circuit Theory
5. Quantum Electronics
6. Integrated Circuits
7. Solid State Device Technology
8. Basic Sciences
9. Military Electronics
10. Information Theory
11. Engineering Education
12. Nuclear Generation of Power

13. Electromagnetics

14. Digital Computers

15. Hybrid and Analog Computers

16. Reliability

17. System Theory

18. Solid State and Gaseous Plasmas

19. Optimal Control

20. Atmospheric Electricity

21. High Voltage DC Transmission of Power

22. Propagation of Pulses (in manmade and natural media)

Instructions for Submission of Papers

Authors are requested to submit three (3) copies of a 300-500 word summary of their paper. In addition, authors should submit four (4) copies of a 35-50 word abstract of their paper. These latter should be typed on separate sheets of paper and should include the name of the paper, the author's name, affiliation, complete return address and telephone number. Carbons or xerox may be used for the copies.

Both the abstract and the summary should be sent on or before December 1, 1965 to:

Dr. L. P. Huelsman

Technical Papers Chairman

1966 IEEE Region Six Conference

c/o Department of Electrical Engineering

University of Arizona

Tucson, Árizona 85721 